\title{
A case of Berardinelli-Seip syndrome presenting with cirrhosis
}

\author{
${ }^{1} \mathrm{H}$ Wimalaratna; ${ }^{2} \mathrm{ASD}$ Nandasiri \\ ${ }^{1}$ Consultant Physician, Teaching Hospital, Kandy, Sri Lanka, ${ }^{2}$ Registrar in Medicine, North Colombo Teaching Hospital, Sri Lanka
}

\begin{abstract}
Berardinelli-Seip congenital lipodystrophy (BSCL) is a rare autosomal recessive disorder of generalised lipoatrophy, characterised by the absence of functioning adipocytes, with lipid being stored in muscles, the liver and the pancreas. The usual presentation is in adulthood, with manifestations of insulin resistance, hypertriglyceridaemia and liver steatosis. Cirrhosis as the first presentation of BSCL in a young adult is rare. We describe a patient with BSCL presenting with cirrhosis. To the best of our knowledge this is the first case of BSCL reported in a Sri Lankan patient.
\end{abstract}

Correspondence to H Wimalaratna
Teaching Hospital
Kandy
Sri Lanka 20000
tel. +00 948123873 I
e-mail
harithlc@sltnet.lk

KEYWORDS Berrardinelli-Seip syndrome, congenital lipodystrophy, generalised lipoatrophy, cirrhosis, insulin resistance

DECLARATION OF INTERESTS No conflicts of interest declared.

\begin{abstract}
A 30-year-old male presented with a two-week history of abdominal distension and progressive lower limb swelling. $\mathrm{He}$ had no symptoms of cardiac failure. No renal symptoms were noted and he had normal urine output. He had no alteration of bowel habit and no history of hepatitis, blood transfusions or sexual promiscuity. There was no preceding history of febrile illness. He was a product of a non-consanguineous marriage and had no family history of liver disease or autoimmune disorders. His only brother had a similar external appearance and died of an unknown illness at the age of eight. Our patient was a teetotaler and a non-smoker.
\end{abstract}

On examination, he was afebrile and not dyspnoeic, pale or icteric. His body mass index was $22 \mathrm{~kg} / \mathrm{m}^{2}$. He had generalised lipoatrophy, predominantly seen in the face, trunk and the limbs (Figure I) Marked acanthosis nigricans was present involving the neck, axillae and the flexural areas of the elbows. His limb muscles were hypertrophied with prominent veins, giving him an athletic appearance. He had bilateral gynaecomastia, absent axillary hair, lower limb pitting oedema and bilateral exposure keratitis. He didn't have features suggestive of autoimmune diseases. $\mathrm{He}$ was haemodynamically stable having a pulse rate of $88 \mathrm{bpm}$ and blood pressure of $140 / 90 \mathrm{mmHg}$ with no evidence of cardiac failure. His abdomen was protuberant with an umbilical hernia and he had ascites with hepatosplenomegaly but without other abdominal masses. Respiratory system and neurological examination were clinically normal.

The full blood count showed a haemoglobin level of II.8 $\mathrm{g} / \mathrm{dl}$ with a mean corpuscular volume (MCV) of $96 \mathrm{fl}$ and the platelets were $53000 / \mathrm{mm}^{3}$. The white cell count was $6200 / \mathrm{mm}^{3}$.

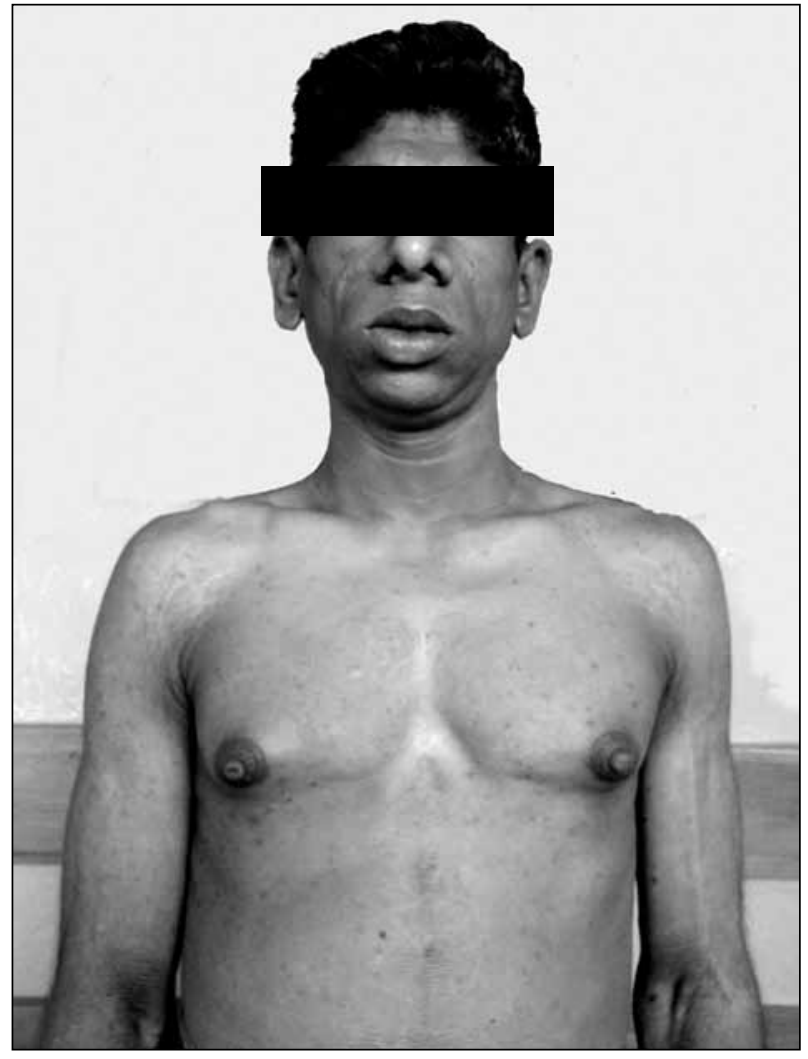

FIGURE I Generalised lipoatrophy, acromegaloid features, acanthosis nigricans involving the neck, axillae, hypertrophied muscles with prominent superficial veins.

Liver functions were deranged with low albumin levels and reversed albumin:globulin ratio (total protein $6.7 \mathrm{~g} /$ $\mathrm{dl}$, albumin $2.4 \mathrm{~g} / \mathrm{dL}$, globulin $4.3 \mathrm{~g} / \mathrm{dL}$ ). Prothrombin time/internal normalised ratio (PT/INR) was I.6. Serum glutamic oxaloacetic transaminase (SGOT), serum glutamic pyruvic transaminase (SGPT), serum bilirubin, alkaline phosphatase and gamma glutamyl transferase 
levels were normal (SGOT 35 iu/L, SGPT 32 iu/L, serum bilirubin $<13 \mu \mathrm{mol} / \mathrm{L}$, alkaline phosphatase $30 \mathrm{iu} / \mathrm{L}$, gamma GT $28 \mathrm{iu} / \mathrm{L})$. An ultrasound scan of the abdomen revealed coarse echotexture with an irregular margin and the surface of the liver with a dilated portal vein of $14 \mathrm{~mm}$ (normal $<10 \mathrm{~mm}$ ). This, along with abnormal synthetic functions of the liver (INR and low albumin level) confirmed the diagnosis of cirrhosis with portal hypertension. A subsequent endoscopy revealed oesophageal varices. Hepatitis B and C, HIV screening and antinuclear antibodies were negative. The iron studies, urinary copper excretion and serum ceruloplasmin levels were normal.

The serum triglyceride level was borderline high (179 $\mathrm{mg} / \mathrm{dL}$ ), while the rest of the lipid profile was normal. His fasting and postprandial blood sugar levels were 4.2 $\mathrm{mmol} / \mathrm{L}$ and $6.5 \mathrm{mmol} / \mathrm{L}$ respectively. His haemoglobin AIC ( $\mathrm{HbAIC}$ ) was $5.6 \%$ and the oral glucose tolerance test result was normal. His electrocardiogram (ECG) and a chest $\mathrm{X}$-ray were normal and the two-dimensional echocardiogram showed concentric left ventricular (LF) hypertrophy. His renal functions were in the normal range and the kidney sizes were normal in ultrasound scans. The patient did not consent to a liver biopsy.

His was treated with furosemide and spironolactone, with routine care for cirrhosis, while being monitored for complications, including assessments for evidence of oesophageal varices. Regular blood pressure checks and frequent monitoring of blood sugar and triglyceride levels were a part of the follow-up for early detection of metabolic derangements. Unfortunately this patient died in another hospital due to probable sepsis a few months after our clinical diagnosis.

\section{DISCUSSION}

Berardinelli-Seip congenital lipodystrophy is a rare autosomal recessive disorder of generalised lipoatrophy. It was first described by Berardinelli in 1954 and was later confirmed by Seip in 1959.' About 200 cases have been reported among various ethnic groups worldwide, with a current prevalence of less than I:12 million.' An extensive literature survey did not reveal any reported case of this syndrome in the Sri Lankan population.

The disorder is characterised by an absence of functioning adipocytes with an inability to store triglycerides in them. This leads to abnormal deposition of triglycerides in other tissues such as muscles, the liver and the pancreas. $^{2}$ As a consequence, the affected individuals develop insulin resistance, hepatic steatosis and skeletal muscle hypertrophy. Generalised lipoatrophy is apparent from birth or from the first month of life. ${ }^{3}$ This, together with hypertrophied skeletal muscles, gives sufferers the appearance of athletes. The prominent superficial veins (phlebomegaly) is due to a lack of subcutaneous fat, especially in the extremities. Insulin resistance will manifest as acanthosis nigricans and diabetes mellitus (DM) by adolescence; it occurs in about $25-30 \%$ of those with BSCL in their twenties. ${ }^{3}$ Hypertriglyceridaemia is common and very high levels result in eruptive xanthomas and recurrent pancreatitis. Hypercholesterolaemia can also occur rarely. ${ }^{2}$ Hepatomegaly develops due to liver steatosis and this occurs in all affected individuals in early life; cirrhosis develops late in the disease course. ${ }^{3}$ Cardiac involvement in this disorder includes hypertrophic cardiomyopathy, concentric or eccentric LV hypertrophy, hypertension and arrhythmias and it can present in infancy or occur in later life. ${ }^{3}$ Other pathologies described in BSCL are psychomotor retardation, mild to moderate cognitive impairment, bone cysts, nephromegaly and nephropathy. Females may have clitoromegaly and hirsutism from birth, precocious puberty or a form of polycystic ovarian disease, with menstrual abnormalities. ${ }^{3}$

The two types of BSCL, type I and 2 have been defined depending on the genes involved. The defect is in the AGPAT2 gene on chromosome $9 \mathrm{q} 34$ in type 1 and BSCL2 on chromosome IIqI3 in type $2 .{ }^{3}$ In type I, only metabolically active adipose tissue is involved. In contrast, type 2 features include greater prevalence of cognitive dysfunction, cardiomyopathy, less cystic bone lesions and involvement of both metabolically active adipose tissue (subcutaneous, intra abdominal, intrathoracic and bone marrow) and mechanical adipose tissue (palms, soles, scalp, retro-orbital). ${ }^{4}$

Clinical diagnosis of BSCL is based on fulfilling the diagnostic criteria. ${ }^{3}$ The presence of three major criteria or two major plus two or more minor criteria, make the diagnosis of BSCL likely.

\section{Major criteria}

I. Lipoatrophy affecting the trunk, limbs and face.

2. Acromegaloid features (gigantism, muscle hypertrophy, advanced bone age, prognathism, prominent orbital ridges, enlarged hands and feet, clitoromegaly and enlarged external genitalia in males).

3. Hepatomegaly.

4. Elevated serum triglyceride.

5. Insulin resistance.

\section{Minor criteria}

I. Hypertrophic cardiomyopathy.

2. Psychomotor retardation or mild to moderate cognitive impairment.

3. Hirsutism.

4. Precocious puberty in females.

5. Presence of bone cysts, polycystic appearance in $\mathrm{X}$-rays in epiphysis of long bones.

6. Phlebomegaly. 
Molecular genetic testing will confirm the diagnosis and differentiate the subtypes. Genetic tests can also be used for carrier testing of at-risk individuals and for prenatal and preimplantation diagnosis. ${ }^{3}$

Management is by improving the insulin resistance and optimising the control of diabetes, dyslipidaemia, hepatic steatosis and its complications. ${ }^{1-4}$ Insulin is the mainstay in treatment of DM and metformin has proven beneficial as it improves the insulin receptor sensitivity, the hepatic steatosis as well as the features of polycystic ovarian syndrome. ${ }^{2,3,5}$ Restriction of dietary fat intake is usually sufficient to maintain normal triglyceride levels but fibrates or fish oil-derived polyunsaturated fatty acids can be used in extreme hypertriglyceridaemia. ${ }^{3}$ Recombinant leptin therapy, though still at the trial level, is supposed to be the most promising treatment as the problem is due to low levels of adipocytokines like leptin and adiponectin. ${ }^{1-3}$ It helps in improving insulin resistance, reducing triglycerides and aids in energy homeostasis.

Death in these patients usually occurs due to haemorrhage from oesophageal varices as a complication of cirrhosis or due to hypertrophic cardiomyopathy and diabetes-related complications.' They survive into young adulthood or to early middle age.

Our patient had generalised lipoatrophy, acromegaloid features, cirrhosis, features of insulin resistance and minimally elevated triglycerides, fulfilling major criteria for the diagnosis of BSCL. As this patient presented with lipoatrophy for the first time at the age of 30 , we excluded acquired causes of lipodystrophy before coming to a diagnosis of BSCL. Though undiagnosed, his brother had a similar lipodystrophic appearance. We suggest that the brother may have had the same condition, with metabolic derangements occurring early in life. This history in a sibling, together with the absence of features of autoimmune disease and panniculitis made it unlikely to be acquired generalised lipodystrophy (Lawrence Syndrome) which presents with almost the same features, but in adult life.

Even though progression of hepatic steatosis to cirrhosis occurs at a later stage it was the presenting symptoms in our patient. Contrary to our expectations, this patient did not have evidence of diabetes but the presence of marked acanthosis nigricans signalled the possibility of developing diabetes in the near future. Cardiomyopathy, bone cysts seen in X-rays, renal impairment and psychomotor retardation were not present but he had unexplained LV hypertrophy.

The clinical picture and the available investigations made the diagnosis of BSCL type I highly likely in this patient. We were unable to perform leptin level tests or the genetic studies which are needed for confirmation due to non-availability in our hospitals. Also, DNA samples were not taken as he died elsewhere, after we had made our clinical diagnosis.

\section{CONCLUSION}

In summary, this patient fulfilled criteria for the diagnosis of BSCL. Even though it usually presents with symptoms related to diabetes or dyslipidaemia, our index case presented with decompensated cirrhosis. To the best of our knowledge this is the first case of BSCL in Sri Lanka and adds to the few cases of this syndrome reported worldwide that presented with cirrhosis. We report this patient to highlight the possibility of BSCL in patients presenting with lipoatrophy, insulin resistance and cirrhosis. We would recommend that regardless of local difficulties concerning access to genetic testing, blood from all patients with a possible genetic condition should be stored for future DNA studies, so that appropriate family counseling can be suitably targeted.

\section{REFERENCES}

I Indumathi CK, Lewin S, Ayyar V. Berardinelli Seip syndrome with insulin-resistant diabetes mellitus and stroke in an infant. Indian J Endocrinol Metab 20I I; 15:S62-4. http://dx.doi.org//0.4I03/22308210.83054

2 Gabbay RA, Khan NR, Arioglu E et al. Generalized lipodystrophy. New York: Medscape; 2012 [cited 2013 Oct 18]. Available from: http://emedicine.medscape.com/article/ I28355-overview

3 Pagon RA, Bird TD, Dolan CR et al (editors). GeneReviews. Seattle: University of Washington; 1993-2013.

4 Roth T, Nair S, Kumar A. Monogenic diabetes secondary to congenital lipodystrophy in a l4-year-old Yemeni girl. J Clin Res Pediatr Endocrinol 2010; 2:I76-9. http://dx.doi.org//0.4274/jcrpe. v2i4. 176

5 Khandpur S, Kumar A, Khadgawat R. Congenital generalized lipodystrophy of Berardinelli-Seip type: a rare case. Indian J Dermatol Venereol Leprol 201I; 77:402. http://dx.doi.org/10.4103/03786323.79740 